\title{
Transporte e Qualidade de Vida
}

Ronaldo Balassiano

Companhia de Transportes Coletivos do Rio de Janeiro - CTC/RJ Transport Studies Group - University of Westminster (PCL)

\section{Sérgio Luiz Chiquetto}

Transport Studies Group - University College London

\section{Ricardo Esteves}

Programa de Engenharia de Transportes - PET/COPPE/UFRJ Bartlett School of Architecture and Planning - University College London

\section{RESUMO}

A questão ambiental está em evidência em todo o mundo. Os impactos ambientais e as possíveis conseqüências de curto, médio e longo prazo decorrentes destes impactos têm sido objeto de atenção nas diversas áreas do conhecimento. Algumas escolas de $1^{\circ}$ grau incorporaram o tema Meio Ambiente aos seus currículos, como forma de familiarizar crianças, desde cedo, com problemas que devem ser atacados hoje e mais seriamente ainda no futuro. Os RIMAs (Relatórios de Impactos no Meio Ambiente) passaram a ser exigência legal a todos os principais projetos de engenharia.

Existem evidências de que os impactos ambientais estão altamente correlacionados com a expectativa de vida humana na Terra. Tanto países considerados "desenvolvidos" quanto aqueles considerados "em desenvolvimento" alegam estar em busca de uma melhor Qualidade de Vida para a população quando promovem ações que de alguma forma impactam o Meio Ambiente. A questão principal a ser resolvida seria ccmo manter e, na maioria dos casos, como melhorar as condições de vida dos povos sem causar danos ao Meio Ambiente, ou em última instância, como minimizar estes danos. 
Entretanto, existem algumas questões que não foram suficientemente exploradas: $O$ que é Qualidade de Vida? Qual o conceito relacionado a esta expressão? Seria possível quantificar Qualidade de Vida? Que fatores contribuem ou estão relacionados com este conceito? Qual a relação entre Transporte e Qualidade de Vida? Dadas a subjetividade e a complexidade embutidas neste conceito, torna-se muito difícil formular respostas precisas para todas estas questões.

O objetivo deste trabalho é, numa primeira etapa, resgatar a discussão sobre a questão da Qualidade de Vida, ressaltando seu caráter subjetivo e as dificuldades envolvidas na avaliação de sua definição. Espera-se, com isto, avançar no sentido de estabelecer uma base conceitual para Qualidade de Vida. Entretanto, o objetivo principal deste trabalho é o de estabelecer o grau de contribuição dos aspectos envolvidos com a oferta de transportes nos níveis de Qualidade de Vida.

Numa abordagem genérica, discute-se a relevância do tema, a relação existente entre Meio Ambiente e Qualidade de Vida, a complexidade e a dificuldade no tratamento desta questão e apresenta-se uma proposta de definição para Qualidade de Vida. No tocante aos Trans. portes, são apresentados os principais fatores que influenciam a Qualidade de Vida, os principais conflitos de interesses e algumas metodologias de avaliação tanto monetária como não-monetária dos impactos ambientais. Ao final, define-se a contribuição dos Sistemas de Transportes na Qualidade de Vida, ao que se desenvolveu um modelo teórico que explicita tal relação.

O modelo proposto pode ser utilizado como instrumento na avaliação da correlação existente entre Transporte e Qualidade de Vida, ainda que apresente algumas dificuldades de aplicação prática graças à considerável subjetividade inerente ao assunto. Tal modelo não esgota a discussão sobre Transporte e Qualidade de Vida, pelo contrário, deve ser considerado um referencial a mais na questão do estabelecimento da correlação existente entre estes fatores. A eficácia na sua utilização estará diretamente ligada ao grau de precisão na determinação de cada um dos fatores que o compõe.

Espera-se com este trabalho, estar contribuindo para um maior entendimento do conceito "Qualidade de Vida" e da relação existente entre Meio Ambiente, Qualidade de Vida e Sistemas de Transportes. 


\section{Introdução}

Com o problema ambiental em evidência no mundo e nas diversas áreas do conhecimento, incluindo a Engenharia de Transportes, nota-se cada vez mais, em textos e discursos, a utilização freqüente da expressão "Qualidade de Vida". Usado muitas vezes de tal forma que chega-se impropriamente a confundi-lo com o conceito de Meio Ambiente ou Impacto Ambiental, o termo "Qualidade de Vida" tornou-se objeto de discussão por parte de técnicos, políticos e interessados em questões ambientais. Passouse a considerar essencial a inclusão deste "indicador" em análises para tomada de decisão. Muitas ações vêm então sendo questionadas ou justificadas com base em argumentações que supõem uma elevação ou deterioração da Qualidade de Vida.

A constatação de que o atual modelo de desenvolvimento ainda não considera de forma satisfatória a questão ambiental, aliada ao fato de que os recursos naturais vem sendo consumidos sem o devido controle, tornou prioritária esta discussão. Verifica-se também uma deterioração generalizada da Qualidade de Vida, prin- cipalmente nos centros urbanos de maior população.

Entretanto, não existe, pelo menos aparentemente, uma definição de consenso para o conceito de Qualidade de Vida, ficando sua utilização sujeita ao sabor das conveniências de cada interlocu. tor, interessado em levar a discussão no sentido da aprovação (ou rejeição) de determinada proposta. O único consenso encontrado parece ser o de que procurar construir uma base conceitual para Qualidade de Vida se trata de uma tarefa de grande dificuldade, graças à subjetividade inerente ao termo.

\section{Objetivos}

Pretende-se, numa primeira etapa, resgatar a discussão sobre a questão da Qualidade de Vida, ressaltando seu caráter subjetivo e as dificuldades envolvidas na avaliação de sua definição. Espera-se, com isto, avançar no sentido de estabelecer uma base conceitual para Qualidade de Vida.

Entretanto, o objetivo principal deste trabalho é o de estabelecer o grau de contribuição dos aspectos envolvidos com a oferta de transportes nos níveis de Qualidade de Vida.

Finalmente, aponta-se algu- 
mas possibilidades de avaliação e medição deste conceito, em relação à questão dos transportes urbanos.

\section{Caracterização Geral}

Segundo as Ciências da Saúde, que aparentemente foram as primeiras a utilizar mais intensivamente este conceito, a qualidade de vida possui algumas dimensões quantitativas, entre as quais a longevidade. Assim, quem tem acesso a uma vida de qualidade, vive mais, pelo menos coletivamente. Desta forma, a literatura desta área registra algumas comparações entre a qualidade de vida de duas ou mais comunidades, utilizando-se como um indicador a média das idades máximas alcançadas pela população.

A literatura também aponta para uma forte correlação entre qualidade de vida e alguns aspectos quantitativos, tais como tamanho da rede de esgotos e quantidade de pessoas atendidas por rede de água. Assim, poderse-ia afirmar, pelo menos intuitivamente, que quanto maior a parcela da população atendida por infraestrutura urbana, maior a qualidade de vida desta comunidade.

$\mathrm{Na}$ verdade, sabe-se, hoje em dia, que este conceito engloba um conjunto muito mais abrangente de fatores, interdependentes ou não, que, de uma forma ou de outra, contribuem para a formação e a eventual alteração desta qualidade.

\subsection{Qualidade de Vida e Meio Ambiente}

Parece claro que existe uma relação bastante forte entre qualidade de vida, meio ambiente e impactos ambientais. Entretanto, uma vez que é igualmente óbvio que não se trata de um mesmo conceito, qual seria, então, a diferença entre estas expressões?

Partindo-se do pressuposto que é importante conhecer esta diferença, a primeira afirmação que se pode estabelecer é que existe um meio ambiente passível de ser impactado, mesmo onde não existe vida. Isto torna o conceito do meio ambiente, e seus eventuais impactos, algo mais abrangente que qualidade de vida.

Pode-se também estabelecer que a vida, à qual a qualidade se refere, se trata da vida humana. Não que as outras vidas, nãohumanas, não tenham a dimensão qualidade, mas é que é sobre a qualidade da vida humana que nós, humanos, temos mais sensibilidade e podemos, portanto, falar com mais propriedade. Isto posto, 
pode-se afirmar que existe um determinado meio ambiente, onde a qualidade de vida é um forte indicador da qualidade ambiental: trata-se do meio ambiente urbano, a cidade, onde as atividades humanas se desenvolvem mais intensamente, o espaço físico está mais concentradamente comprometido com estas atividades e, afinal, é onde se encontra a maior quantidade de vidas humans. Por se tratar, sobretudo, do ambiente cuja a qualidade é mais sensivelmente impactada pelos sistemas de transporte, tornou-se alvo de concentração neste trabalho.

\subsection{Complexidade e Diversidade de Percepção}

A qualidade de vida pode ser influenciada por diversos fatores e sua percepção pode ser diferente dependendo de quem, onde e quando está sendo analisada. Uma vez que é considerada não apenas como um conceito subjetivo, mas também como a conseqüência da conjugação de diversos fatores, a qualidade de vida pode apresentar algumas variações:

- de uma pessoa para outra: diferentes indivíduos, com aspirações e escalas de valores diversos, estéticas e formas distintas de avaliação, podem ter per- cepções diversas de qualidade de vida;

- ao longo do tempo: os indivíduos tendem a alterar sua escala de valores ao longo de sua vida. A percepção de qualidade de vida depende também do estado geral e da atividade que está sendo desenvolvida pelo indivíduo num momento específico;

- de uma comunidade para outra: a percepção de qualidade de vida pode apresentar profundas diferenças entre comunidades de culturas distintas. Contudo, mesmo quando a dimensão cultural não é relevante, a percepção de quali. dade de vida para uma comunidade pode ser bastante diferente da sensibilidade de pessoas pertencentes a outra comunidade. O que é aparentemente considerado uma vida de qualidade para uma comunidade indígena, por exemplo, muitas vezes contradiz os conceitos criados por uma sociedade capitalista. A mesma comparação pode ser feita em relação a comunidades urbanas e rurais;

- de um grupo ecológico para outro: o que é considerado favorável ao aumento da qualidade de vida humana pode 
significar uma redução para outras espécies da fauna ou flora, por exemplo.

\subsection{Proposta de Definição}

Qualidade de vida pode ser entendida como a resultante das condições materiais, culturais, espirituais e outras, que possibilitam, ou ao menos favorecem, a conquista de um estado de bem estar geral. Assim, qualidade de vida pode ser expressa através de um somatório de fatores, devidamente ponderados quanto à sua importância relativa, que contribuem para aumentar ou diminuir o grau de satisfação de um indivíduo ou comunidade.

Desta forma, pode-se intuir que quanto melhor a qualidade de vida (de um indivíduo ou de uma coletividade), maiores são as possibilidades de se atingir níveis mais altos de bem estar (pessoal ou coletivo). Conclui-se, então, que existe uma relação direta entre qualidade de vida e bem estar ou satisfação.

\section{Transporte e Qualidade de Vida}

A grande maioria das atividades econômicas depende do transporte de bens e pessoas. Os sistemas de transportes desempenham, portanto, um papel impor- tante em relação ao estilo de vida das pessoas e conseqüentemente em relação ao seu comportamento.

É inegável que melhorias no sistema de transporte aumentam a mobilidade e a acessibilidade de uma população, favorecendo, desta forma, sua qualidade de vida.

Por outro lado, estas melhorias podem também causar impactos negativos, que afetam a qualidade de vida desta mesma popula̧̧ão. O tráfego de veículos pode provocar alterações ambientais tais como poluição do ar, vibração, ruído, intrusão visual, segregação, comprometimento da segurança e congestionamento de tráfego. Outros efeitos podem ocorrer: problemas à saúde humana, perturbação, desconforto, irritação, frustração, medo, intimidação ou abalo psicológico, fadiga do motorista, exposição a risco, acidentes e perda de vidas, perda de tempo de lazer, atrasos e aumento de custos, degradação dos níveis de serviço, necessidade de manutenção de prédios e de materiais danificados, contribuição para o efeito estufa e mudanças no uso, valor e ocupação do solo.

O número de acidentes pode ser considerado como um típico 
indicador da qualidade de vida com relação ao sistema de transporte. Entretanto, para se avaliar as implicações dos transportes na qualidade de vida, várias outras externalidades negativas causadas por determinado sistema, devem também ser consideradas de alguma forma em tal avaliação.

\subsection{Conflitos de Interesse}

São vários os conflitos de interesses existentes em relação a qualidade de vida de segmentos diversos, quando esta questão se relaciona ao transporte. De uma forma ambígua, um sistema de transportes pode melhorar a qualidade de vida de parte de uma comunidade e trazer ao mesmo tempo perdas para outra parte desta mesma comunidade. Existem ainda conflitos de interesses entre viajantes e residentes ou trabalhadores (usuários e não usuários). Ao se aumentar a mobilidade de alguns, outros poderão ser afetados de forma diversa. Uma estrada de ferro ou rodovia expressa, por exemplo, pode aumentar as facilidades de transporte para os usuários, mas ao mesmo tempo promover a segregação da comunidade local. É também possível que algumas propriedades sejam valorizadas (monetariamente) enquanto outras tenham seus preços reduzidos. Alguns podem se sentir beneficiados enquanto outros se julgam prejudicados.

Não obstante, em alguns casos a opinião de técnicos não coincide com a da comunidade local, o que torna fundamental o envolvimento desta comunidade no processo de avaliação e no desenvolvimento de dispositivos capazes de conduzir à melhor solução possível. Desta forma, uma política de transportes deve ser questionada em termos de melhorar ou não a qualidade de vida de uma comunidade, considerando todos os seus segmentos.

\subsection{Dificuldades de Avaliaçäo}

Embora não se conheça uma escala capaz de "medir" a qualidade de vida, é possível se obter uma avaliação desta qualidade através dos impactos ambientais provocados pelos sistemas de transportes. Entretanto, não é fácil compreender-se o problema ambiental como um todo dado que, acima de tudo, é essencialmente subjetivo em natureza e envolve interações complexas entre vários fatores. Alguns autores, como Ackoff (1976), chegam mesmo a questionar a validade de se quantificar qualidade de vida.

Enquanto alguns impactos à 
saúde, flora, fauna e deterioração de materiais são facilmente identificáveis, muitos impactos se dão no campo de distúrbios, aborrecimentos, risco, medo, intimidação, perturbação ou efeitos psicológicos. Se, por um lado, os fatores quantificáveis apresentam enormes dificuldades para serem somados em uma mesma escala, o que dizer sobre a parte subjetiva da avaliação?

Da mesma forma, torna-se muito complexo avaliar o custo financeiro de efeitos decorrentes dos impactos gerados pelos sistemas de transportes, como o efeito-estufa, a degradação de fachadas e outros materiais, pessoas adoecendo ou até morrendo e ainda despesas com os serviços de saúde. Algumas metodologias foram desenvolvidas para a determinação destes custos, porém com resultados sempre questionados devido à subjetividade metodológica relacionada a cada processo. A degradação ambiental passada e presente será uma herança para futuras gerações, o que adiciona a complexidade de como avaliar monetariamente um efeito que somente ocorrerá no futuro e, acima de tudo, se apresentar características de irreversibilidade.

Não é muito díficil avaliar o impacto (positivo ou negativo) que uma variável específica pode exercer em termos de qualidade de vida. Por exemplo, se o nível de ruído, vibração e número de acidentes aumentarem na vizinhança de uma rodovia, como conseqüência da implementação de um programa visando aumentar a velocidade e fluidez do tráfego, pode-se esperar que a qualidade de vida da comunidade local tenha diminuído. Contudo, se os demais impactos causados compreendem também efeitos positivos, como diminuição da poluição do ar, aumento das condições de acessibilidade e redução dos tempos de viagem e custos, a avaliação global passa a ser consideravelmente mais complexa.

Comparar diferentes situações envolvendo aspectos distintos influenciadores da qualidade de vida também é uma tarefa árdua. Pode não ser trivial a comparação de particularidades entre pessoas vivendo em diferentes localidades e sujeitas a distintas condições e definir qual tem o mais alto padrão de qualidade de vida.

Tudo se tornaria mais fácil se as diferenças existentes entre situações distintas fossem evidentes. Ainda, essas dificuldades não fariam sentido se cada fator 
pudesse ser pesado ou recebesse um valor (talvez em termos monetários) em uma escala comum.

Análises custo-benefício de impactos no meio ambiente em um sistema como um todo tem sido realizadas; porém estão sujeitas a uma série de limitações e imprecisões.

\subsection{Técnicas de Avaliação de Impactos Ambientais}

Partindo-se do pressuposto que os impactos ambientais são importantes indicadores das condições de vida de uma comunidade, para se avaliar a contribuição dos transportes na qualidade de vida, primeiramente é necesário se avaliar os impactos produzidos pelos transportes nesta comunidade.

Tradicionalmente, são conhecidas abordagens qualitativas e quantitativas para avaliação de impactos ambientais. Ambas consideram os diversos fatores ambientais em uma escala (qualitativa ou quantitativa) de valores e atribuem pesos próprios a eles de acordo com a intensidade da degradação. Contudo, a literatura divide as técnicas de avaliação em duas categorias mais específicas, que são as técnicas monetárias e as não-monetárias. Por lidarem com parâmetros subjetivos e esta- rem sujeitos a uma série de limita६̧ões, nenhuma delas tem sido suficientemente explorada. Embora essenciais em grande parte das avaliações de planos e políticas de transporte, as técnicas de avaliação de impactos ambientais ainda estão num estágio muito recente de desenvolvimento e aceitação.

As técnicas não-monetárias, de uma forma geral, atribuem graus de importância relativa entre os diversos impactos, enquanto que as monetárias atribuem valores em moeda corrente (unidade comum no mercado) a cada impacto. As principais técnicas de avaliação serão brevemente discutidas a seguir, mas para uma abordagem mais completa sobre este assunto recomenda-se a leitura do trabalho desenvolvido pelo Rendel Planning and Environmental Appraisal Group (1992).

\subsubsection{Técnicas Não-Monetárias}

Assim como as monetárias, as técnicas não-monetárias tentam estabelecer bases comuns na avaliação de impactos ambientais. Estas técnicas transformam as informações sobre os impactos em um conjunto de números de forma a obter-se uma pontuação e determinar, por exemplo, a melhor 
solução na comparação entre projetos. Pode-se ainda produzir uma classificação entre diferentes alternativas após as devidas comparações.

As técnicas não-monetárias mais conhecidas podem ser agrupadas em 5 formas distintas: técnicas lexicográficas, técnicas de maximização de consenso, técnicas agregacionais, técnicas gráficas e técnicas de concordância (Rendel Planning and Environmental Appraisal Group, 1992).

As técnicas lexicográficas assumem que os critérios de avaliação podem ser ordenados por grau de importância. São conhecidos dois tipos de modelos de avaliação: o primeiro rejeita projetos que não alcancem um padrão mínimo estabelecido. O segundo seleciona o projeto com a maior pontuação no critério julgado de maior importância.

As técnicas de maximização de consenso foram desenvolvidas para identificar prioridades e visóes de diferentes grupos da sociedade baseados na percepção da importância de cada um dos critérios.

Técnicas gráficas e agregacionais assumem que pontuações padronizadas podem ser dadas para alternativas quando avaliadas por diferentes critérios. As técnicas gráficas assumem que os valores atribuídos para projetos alternativos podem ser plotados em gráficos multi-dimensioais, enquanto que nas técnicas agrega. cionais, cada projeto recebe uma pontuação para cada um dos critérios. Esta pontuação é então padronizada e multiplicada pelo peso de cada critério antes da agregação final para obter-se um resultado ou índice.

Na utilização de técnicas de concordância são atribuídos pesos aos diversos critérios, sofrendo variações de forma a obter-se uma análise de senbilidade dos impactos.

Todas as metodologias apresentam dificuldades reais de utilização prática. Não é fácil a operacionalização destas técnicas como forma de avaliar a melhor alternativa entre dois projetos. Entretanto, alguns aspectos destas técnicas podem ser utilizados como um instrumento auxiliar na avaliação de impactos de projetos de transportes. As maiores críticas a estas técnicas recaem na problemática de atribuir-se pesos aos diversos critérios de avaliação, uma vez que estes pesos podem variar com o tempo, local, escala, grupo e tipo de proposta.

\subsubsection{Técnicas Monetárias}


A escala quantitativa mais conveniente para medir os impactos ambientais é a monetária. A atribuição de valores monetários para impactos ambientais é considerada um instrumento importante para estudos envolvendo análise custo-benefício e também na avaliação de investimentos para implementação de planos, programas e políticas de transportes.

A idéia de creditar valores quantitativos ou custos sociais monetários aos fatores ambientais é relativamente recente. No Reino Unido, a mais abrangente e sofisticada tentativa de atribuir valores monetários a aspectos ambientais foi feita pela Comissão Roskill (1970), por ocasião da avaliação de custos e benefícios provenientes dos estudos sobre a implantação do terceiro aeroporto em Londres. Logo em seguida, Baumol e Oates (1971) propuseram o uso de alguns standards e preços para a proteção do meio ambiente. Sugerem, ainda, que o valor monetário para externalidades negativas pode ser encontrado através do preço para o uso privado de recursos sociais ou do preço do prejuízo ou dano marginal gerado. Alguns trabalhos, como por exemplo Walters (1975) e Pearce (1976) fizeram uma extensiva revisão sobre 0 assunto e enfatizaram sua dificuldade, mas, acima de tudo, sua importância. Langdon (1978) reporta os resultados de uma ampla pesquisa sobre a perturbação do ruído de tráfego, executada em Londres, na qual os entrevistados foram convidados a avaliar o valor monetário de um ambiente calmo e tranqüilo. Hansson (1991) reporta a introdução de instrumentos econômicos na política ambiental da Suécia, que atribui taxa para a emissão de poluentes. Barde e Pearce (1991) editaram uma coleção de textos que refletem as experiências de seis países em atribuir valores ao meio ambiente. Rendel Planning and Environmental Appraisal Group (1992) apresenta uma revisão detalhada sobre os vários métodos existentes para avaliações monetária e não-monetária de aspectos ambientais.

Entretanto, o estabelecimento de parâmetros que incluem a dimensão quantitativa em estudos de transporte sempre foi complexo. Não tem havido progresso significativo no campo da gerência ambiental para a área de transportes, embora muitas tentativas tenham sido feitas para se deduzir um valor próprio, preço ou custo para os impactos negativos do trá- 
fego de veículos. Na prática, quando disponiveis, as técnicas raramente identificam claramente o impacto, e com muito maior dificuldade atribuem valores monetários a ele. Na verdade, como Pearce (1976) argumenta, nenhum dos métodos conhecidos até então consegue oferecer resultados confiáveis e suficientemente precisos.

Mesmo assim, diversas técnicas existentes continuam em fase de desenvolvimento e cada vez mais têm sido utilizadas na tentativa de se avaliar os impactos ambientais com um embasamento científico mais apropriado. Algumas das técnicas de avaliação mais relevantes são brevemente abordadas a seguir.

As técnicas para avaliação do custo de proteção ambiental se baseiam em estimativas do preço do prejuízo causado por um efeito degradante. Estas estimativas podem ser realizadas através do somatório de todas as contribui̧̧ões parciais que geram qualquer prejuízo, dano ou estrago em termos monetários, ou em termos dos custos necessários para eliminar ou mitigar um impacto, como implantação de catalisadores e barreiras contra o ruído. O principal problema da implantação desta técnica recai exatamente em estabelecer bases para avaliar monetariamente os danos ambientais, em virtude do número e natureza das atividades, pessoas, bens e outros diversos fatores envolvidos serem tão grandes que tornam qualquer estimativa sujeita a muitas incertezas. O pre. juízo causado envolve danos à saúde, materiais e bens, custos estéticos e pessoais. Por isto, é necessário um conhecimento bastante aprofundado da questão para se considerar estes custos de forma eficaz.

As técnicas hedônicas de avaliação procuram estimar valores ambientais, que geralmente não têm preços de mercado, através de mudanças nos valores de propriedades, por conseqüência da ocorrência de impactos ambientais. Os dois principais problemas em relação a esta técnica são, primeiro, que torna-se difícil analisar a influência de mais de um impacto ao mesmo tempo e segundo, a necessidade de dados relativos aos níveis dos impactos ambientais e de preços das propriedades afetadas.

Outra forma de se atribuir valores monetários aos impactos provenientes do tráfego de veículos é através da avaliação da preferência dos usuários ou comunidade interessada. A técnica mais 
utilizada se baseia em simulações de mercado, onde os respondentes expressam suas valorações monetárias hipotéticas a respeito de mudanças ambientais. Em tal pesquisa, os entrevistados devem avaliar sua disposição em pagar um determinado valor monetário mínimo pela prevenção, eliminação ou redução de um determinado impacto ambiental até um certo nível desejado. Este método para atribuir valores monetários a parâmetros qualitativos foi um dos primeiros a ser adotado nos Estados Unidos, originalmente para dar valor a economia de tempo de viagem (Howe, 1971). Diversas variações desta técnica foram desenvolvidas e aplicadas, de acordo com a especificadade de cada problema (Dixo et al., 1988). A maior dificuldade é que categorias subjetivas derivadas de pesquisas de opinião, tais como insatisfação e perturbação, são sujeitas a todo tipo de incerteza e sempre permanecerão, em grande parte, em temos subjetivos. Não se pode esperar que os entrevistados tenham uma idéia razoavelmente precisa de tais valores se eles provavelmente nunca foram submetidos a semelhante tarefa.

4.4 Proposta para Avaliação da
Contribuição dos Transportes na Qualidade de Vida

Como dito anteriormente, o impacto positivo mais importante causado pelo transporte é a melhoria das condições de acessibilidade. Contrariamente, as externalidades negativas são compreendidas dentro de uma ampla variedade de impactos tais como poluição do ar, ruído, vibração, intrusão visual, alterações no ambiente dos pedestres, redução da segurança e segregação urbana.

A contribuição relativa à oferta de transportes na qualidade de vida de uma certa comunidade pode ser compreendida como o valor teórico obtido a partir da soma de todas as externalidades positivas e negativas percebidas pela comunidade, devidamente ponderadas pelo número de pessoas afetadas e pelo grau relativo de importância ou sensibilidade delas em relação a cada um dos impactos verificados. Assim:

$Q V_{t}=a_{1} \cdot b_{1} \cdot I_{1}+a_{2} \cdot b_{2} \cdot I_{2}+\ldots+a_{n} \cdot b_{n} \cdot I_{n}$

$Q V_{t}=\sum_{x=1}^{n} a_{x} \cdot b_{x} \cdot I_{x}$

onde: 
$\mathrm{QV}_{\mathrm{t}}=$ Contribuição do Trans porte na Qualidade de Vida.

$I_{x}=$ Impacto Ambiental $x$. Deve ${ }^{x}$ ser precedido pelo sinal "+", se o impacto for considerado positivo ou pelo sinal "-" se o impacto for negativo;

$a_{x}=$ peso relativo a importância, intensidade ou sensibilidade atribuída ao Impacto $x$. Este coeficiente pode ser encontrado através de técnicas tanto monetárias como não-monetárias para atribuir valores a cada impacto ambiental, como as relatadas no item 4.3, embora reconhecidas as dificuldades de aplicação de ambas.

$b_{x}=$ número de pessoas afetadas pelo Impacto x. Para isto, inicialmente, a área de influência tem que ser identificada.

Como pode ser deduzido pela formulação matemática, para cada trecho de uma malha viária, a degradação relativa a cada impacto deverá ser ponderada por sua intensidade e pelo número de pessoas afetadas. Assim, a escala resultante de qualidade de vida pode ser associada a gama de padrões de qualidade, que representaria as condições ambientais no local.

Alternativamente, cada impacto poderia ser desmembrado nos diversos efeitos dele decorrentes e a cada efeito uma ponderação pode ser feita isoladamente, o que, se por um lado aumenta a dificuldade de aplicação, por outro lado tende a melhorar a precisão dos resultados.

De uma forma ou de outra, uma estimativa quantitativa para qualidade de vida pode ser formulada para uma determinada área, submetida a um conjunto específico de impactos causados pelos sistemas de transportes existentes. Fica claro que tal estimativa é baseada na definição dos indicadores ou critérios levados em consideração e dos pesos atribuídos a cada um deles. Isto indica que qualquer avaliação deverá ser suficientemente cautelosa para considerar todos os possíveis fatores envolvidos e produzir uma boa estimativa dos pesos relativos a cada fator; caso contrário os resultados podem ser não realísticos.

\section{Conclusões}

Um modelo teórico para avaliação da contribuição dos transportes na qualidade de vida serve como instrumento para tomada de decisão, por possibilitar:

- comparação de situações antes/depois da implementação de um plano de tráfego/transporte e suas implicações na qualidade de vida; 
- análises de impactos a serem causados por projetos futuros;

- comparações, embora relativamente grosseiras, da qualidade de vida de diferentes grupos ou comunidades.

Cada um dos termos que compõe o modelo apresenta dificuldades específicas em relação à identificação e valoração. A primeira dificuldade reside na identificação dos possíveis impactos gerados pelos sistemas de transportes. A segunda está na complexidade do estabelecimento de bases comuns na determinação dos valores e importância relativa de cada impacto. Finalmente, embora possa não parecer em uma primeira análise, não é trivial limitar a região impactada e identificar a parcela da população afetada por estes impactos.

O modelo apresentado não esgota a discussão sobre Transportes e Qualidade de Vida. Pelo contrário, o modelo deve ser considerado um referencial a mais na questão do estabelecimento da correlação existente entre estes fatores.

É importante notar que a avaliação dos impactos provocados por um mesmo sistema de transportes sobre duas regiões diferentes, muito provavelmente não apresentará os mesmos resultados, em que pese a acurácia da avaliação, devido às dificuldades, subjetividades e especificidades já discutidas neste trabalho.

Ainda, é recomendável que cada um dos aspectos aqui abordados sejam levados em consideração quando na análise da questão para que o modelo possa produzir resultados satisfatórios em uma análise da contribuição dos transportes na qualidade de vida.

Finalmente, considera-se importante o incentivo à pesquisa nesta área, de forma a encontrarse valores quantitativos mais realísticos para os parâmetros subjetivos que envolvem a avaliação de qualidade de vida.

\section{Referências Bibliográlicas}

ACKOFF, R.L. (1976) - Does Quality of Life have to be Quantified?, in Operational Research Quaterly, vol.27, n.2, Pergamon Press.

BANISTER, D. and BUTTON. (1992) - Transport, the Environment and Sustainable Development (to be published).

BARDE, J.P. e PEARCE, D.W. (1991) - Valuing the Environment, Six Case Studies, 
Earthscan Publications Limited.

BAUMOL, W.J. and OATES, W.E. (1971) - The Use of Standards and Prices for Protection of the Environment, The Swedish Journal of Economics, Vol.13, n.1., pp 4254.

BAUMOL, W.J. and OATES, W.E. (1975) - The Theory of Environmental Policy Externalities, Public Outlays and the Quality of Life, Prentice-Hall.

BELLIA, V. e BIDONE, E. (1990) - Rodovias, Recursos Naturais e Meio Ambiente (no prelo).

DEPARTMENT OF TRANS PORT (1983) - Manual of Environmental Appraisal, HMSO.

DIXON, J.A. et al. (1988) Economic Analysis of the Environmental Impacts of Development Projects, Earthscan Publications Limited.

DRENOWSKI, JAN (1975) On Measuring and Planning The Quality of Life, Publications of the Institute of Social Studies, Mouton-The Hague. FREEMAN, A. (1979) - The Benefits of Environmental
Improvement: Theory and Practice, Resources for the Future, University Press.

HALL, J.F. (1974) - Indicators of Environmental Quality and Life-Satisfaction: a Subjective Approach, Social Science Research Council, Survey Unit. UK.

HANSSON, L. (1991) - The Pricing of Air Pollution in the Swedish Transport Policy, Transportation Research Board's 70th Annual Meeting, January 13-17 1991, Washington DC.

HOWE, J.D.G.F. (1971) - The Value of Time Savings from Road Improvements: A Study in Kenya, Transport and Road Research Laboratory LR 372.

KIND, P. (1988) - The design and Construction of Quality of Life Measures, Institute for Health Economics, University of York.

LANGDON, F.J. (1978) Monetary Evaluation of Nuisance from Road-Traffic Noise: An Exploratory Study, Environment and Planning $A$, Volume 10, pp 1015-1094.

PEARCE, D.W. (1976) - Environmental Economics, Longman, London. 
PEARCE, D.W. (1990) - The Valuation of Transport-Induced Environmental Costs and Benefits, Seminar on Longer Term Issues in Transport Economics.

PEARCE, D.W.; MARKAN DYA, A. and BARBIER, E.B. (1989) - Blue Print for a Green Economy, Earthscan Publications.

RENDEL PLANNING AND E N V IR O N M E N T A L APPRAIS AL GROUP (1992) - Environmental Appraisal: A Review of Monetary Evaluation and Other Techniques, Transport Research Laboratory CR 290.
ROSKILL COMISSION (1970)

- Comission on the Third London Airport, Papers and Proceedings, Volume II, HMSO.

SACTRA, Standing Advisory Committee on Trunk Road Assessment (1992) - Assessing the Environmental Impact of Road Schemes, HMSO.

WCED, World Comission on Environment and Development (1987) - Our Common Future, Oxford University Press.EPWALTERS, A.A. (1975) - Noise and Prices, Clarendon Press, Oxford. 